\title{
Surgical Management of Thyrotoxicosis in Children
}

\author{
By Edward S. Tank, George E. Bacon, and George H. Lowrey
}

Q INCE THE INTRODUCTION of thyroid blocking agents 20 years ago $D$ there has been a continuing controversy over the best form of treatment for thyrotoxicosis in children.

In terms of ease, expense, and short-term safety there is no doubt that radioactive iodine is superior to either subtotal thyroidectomy or the administration of antithyroid drugs. The potential hazard of radioactive iodine are the induction of thyroid cancer, leukemia, genetic damage, and permanent hypothyroidism. The magnitude of these risks in childhood is unknown and this uncertainty has prompted most centers including ours to restrict this form of therapy to adults and the rare instances in children when the use of the other forms of treatment is contraindicated. Some very well-respected clinicians are using radioiodine in children. Their long-term results will either vindicate or abolish the fears of the majority. Since long-term followup will be required to determine the answer to the most threatening fears, we must wait and decide among the other modalities at hand.

Management of this disease process with drugs requires a prolonged period of administration and close supervision by the physician. At best only a $60-75$ per cent incidence of permanent remission has been achieved. It has been the feeling of our institution that subtotal thyroidectomy would be the preferred form of therapy if the euthyroid state could be consistently attained with an absence of mortality and a minimum of morbidity. ${ }^{1}$ Twenty-three years of experience at the University of Michigan Medical Center with bilateral subtotal thyroidectomy for thyrotoxicosis in children was reviewed in order to make this decision.

\section{C anical Materia'}

Since 1945, 64 infants and children under 13 years of age with a proven diagnosis of thyrotoxicosis have been evaluated at the pediatric Endocrine Clinic. Fifty-two were females and 12 were males. Age distribution at the onset of symptoms is illustrated in Table 1 . Seventy-five per cent of the patients fell into the 8 to 13 year range. The mean age of all patients was 9.5 years. Common presenting signs and symptoms were nervousness, weight loss, and emotional disturbances. Over 50 per cent of the patients presented with exophthalmos, tachycardia, and systolic hypertension. There were 2 infants

From the Departments of Surgery and Pediatrics, University of Michigan Medical Center, Ann Arbor, Michigan 48104.

Edwaro S. TANk, M.D.: Assistant Professor of Surgery, Section of General Surgery, Department of Surgery, University of Michigan Hospital. GeuhGe E. Bacon, M.D.: Assistant Professor of Pediatrics, Department of Pediatrics, University of Michigan Hospital. George H. Lowrey, M.D.: Professor of Pediatrics, Department of Pediatrics, University of Michigan Hospital. 
Table 1.-Age Incidence of Thyrotoxicosis (Mean Age 9.5 Years)

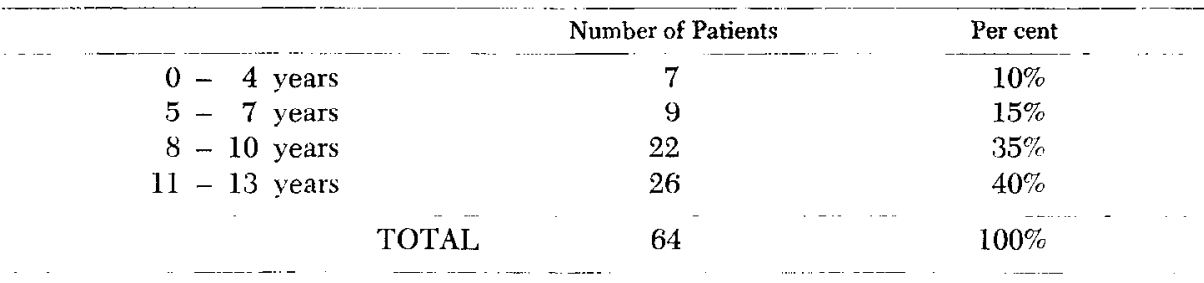

born with thyrotoxicosis: both of these infants had exophythalmos and thyroid enlargement. Failure of the children to gain weight was the primary manifestation of the disease. All clinical impressions were substantiated by serum protein bound iodine determinations or radioactive uptake studies.

Forty-five children had bilateral subtotal thyroidectomy. The parents of 7 children elected to have operative procedure performed at hospitals nearer their place of residence. Ten children received medical therapy. One of the 2 newborn infants had spontaneous resolution of the disease process without medication. One responded to medical therapy.

The patients who were to undergo operative therapy were prepared according to a general plan. Propylthiouracil was given in divided daily doses for an average of 6 weeks prior to operation. The daily dose varied from 150 to $300 \mathrm{mg}$. daily and was always given at 6 to 8 hour intervals to maintain a constant blood level. Iodine in the form of Lugol's solution was given for the 2 weeks prior to operation in single daily doses to reduce vascularity of the gland. The amount given was usually 5 drops. A patient was considered to be well prepared for surgery if he had shown considerable weight gain, a decrease in pulse rate to the normal level for his chronologic age, and clinical improvement such as decreased irritability and emotional lability. A normal protein bound iodine determination was also obtained prior to the administration of iodine.

\section{Surgical Technic}

Bilateral subtotal thyroidectomies were carried out by members of the permanent and resident staff. The technic used was that perfected by Frederick Coller. 2 A Kocher incision is made approximately one finger's breadth above the upper borders of the inner ends of the clavicles. The platysma is divided transversely. The upper Hap of skin and platysma is readily dissected upward from the deep fascia to just above the thyroid notch with a minimum of bleeding by means of a piece of gauze over the gloved finger. The lower flap is developed only to a limited extent. The deep cervical fascia is divided in the midline to a point slightly above the thyroid notch, thus exposing the isthmus of the thyroid. This permits lateral retraction of the sternohyoid and sternothyroid muscles and exposes the lobes of the thyroid gland. The suspensury fascia of the thyroid is then divided above the isthmus and the upper tracheal rings exposed as a landmark for future reference. If a pyramidal lobe is found at this point it is completely freed. After exposure of the tracheal rings above the isthmus the cricothyroid space lying between the medial aspect of the upper pole and the thin fascia covering the cricothyroid muscle is defined by blunt dissection. This space can be developed without fear of injury to the upper thyroid vessels and permits complete separation of the superior pole from the larynx, thus avoiding injury to the branches of the superior laryngeal nerve as they enter the larynx and the cricothyroid muscle. The superior thyroid vessels are now well exposed and are ligated 
Table 2.-Complications in Forty-Five Bilateral Subtotal Thyroidectomies

\begin{tabular}{ll} 
Laryngeal edema & \\
$\quad$ requiring temporary tracheostomy & 2 \\
Vocal cord paralysis & 1 \\
$\quad$ Temporary & 2 \\
$\quad$ Permanent, asymptomatic & 2 \\
Hypoparathyroidism & 2 \\
mild, controlled by oral medication & 2 \\
Enlargement of remnant & 0 \\
\hline
\end{tabular}

and divided. This permits dislocation of the upper lobe of the thyroid anteriorly. At this point the lateral vein is divided. It is always possible to deliver the lower lobe of the gland into the wound even if a great portion of it lies below the sternum because of its lack of anatomic attachments. Hemostats are now placed on the lateral surface of the lobe to outline the area for resection. A thin layer of thyroid tissue is left with the posterior thyroid capsule to protect the parathyroid glands and the recurrent laryngeal nerve. A volume of tissue weighing approximately $500 \mathrm{mg}$. is left on each side in children. This dissection is carried out from the lateral side and is guided as the trachea is approached. The isthmus in its entirety is dissected carefully from the trachea. Careful hemostasis is then obtained by suture ligature. The remaining thyroid tissue is not folded over on itself or sutured over the trachea because the danger of producing torsion of the recurrent laryngeal nerves. Meticulous hemostasis obviates draining of the wound. The prethyroid muscles are sutured in two layers over the trachea. The deep cervical fascia is then reapproximated. The platysma is then reconstituted with buried $4-0$ white silk sutures, and the skin is closed with interrupted 4-0 nylon. Half of the sutures are removed on the second day following operation and the remaining ones are taken out 24 hours later.

\section{RESUL'TS}

Using this technic surgical complications have been minimized (Table 2). Two children required temporary tracheostomy because of postoperative laryngeal edema. Unilateral vocal cord paralysis occurred in 3 patients. In one the palsy was transitory and in two others it was permanent but asymptomatic. Mild hypoparathyroidism occurred in 2 cases. Both of these patients are easily controlled on oral medication. Two patients experienced enlargement of the thyroid remnant. These children were euthyroid by clinical and laboratory parameters. The remnant regressed on administration of desiccated thyroid. There was no operative mortality.

Thirty-five of the 45 children ( 77 per cent) undergoing bilateral subtotal thyroidectomy have been followed for at least 2 years. Seven children were followed for 10 years, 18 for 5 years, and 10 for 2 years. In over 75 per cent of the cases serum protein bound iodine determinations and/or radioactive iodine uptake studies were performed and confirmed the clinical impression. Recurrent hyperthyroidism did not occur. Only two children ( 5 per cent) had evidence of hypothyroidism (Table 3 ). There is no evidence of a progressive increase in hypothyroidism as the follow-up interval increased.

\section{Discussion}

The 2 infants born with thyrotoxicosis are an interesting facet of the disease. Both mothers had thyrotoxicosis. A hormone termed long-acting thyroid 
Table 3.-Incidence of Postsurgical Hypothyroidism

\begin{tabular}{|c|c|c|}
\hline Follow-up Interval & No. of Patients & Hypothyroid \\
\hline 10 years & 7 & 0 \\
\hline 5 years & 18 & 1 \\
\hline \multirow[t]{2}{*}{2 years } & 10 & 1 \\
\hline & TOTAL & $2(5 \%)$ \\
\hline
\end{tabular}

stinulator (LATS) is found in serum of the mother and crosses the placenta and leads to fetal hyperthyroidism. ${ }^{3}$ This also explains the self-limited nature of the process without medication and its spontaneous resolution within 1 to 3 months after birth. These children usually present with prematurity, goiter, and exophthalmos. Congestive heart failure is a common presenting feature.

The potential risk of radioactive iodine administration-thyroid cancer, leukemia, and genetic damage-do not warrant its use except in unusual circumstances. Starr ${ }^{4}$ reported a 58 per cent incidence of post-treatment hypothyroidism in 73 children given $\mathrm{I}^{131}$ for thyrotoxicosis. The choice of therapy at the present time falls between drugs (propylthiouracil or other thiourea derivatives) and operation (bilateral subtotal thyroidectomy).

The modern antithyroid drugs found many supports following their introduction in the mid 1940's. Encouraging case reports appeared, and some hospital centers began to use drug therapy almost exclusively. At least one contributing element leading to the acceptance of this form of therapy was the fact that the pediatrician was not forced to share the responsibility of the treatment of the patient with the surgeon.

Sufficient time has now passed to allow critical evaluation of drug therapy. Comparison of results is difficult because of the varying duration of the follow-up period, and because some of the larger studies include adults as well as children. Information is presented by Solomon and co-workers ${ }^{5}$ indicating that the incidence of relapse increases with the passage of time. In their series of 101 patients (mostly adults) nearly 70 per cent were in remission one year after cessation of the medical therapy. This figure had dropped to approximately 60 per cent after 2 years, and only 55.5 per cent were considered euthyroid after 4 years or more. These authors compiled data from 11 other series, each including 20 or more cases, and obtained an essen. tially identical cure rate.

In studies confined to the pediatric age group, Kunstadter and Stein ${ }^{6}$ reported at best a remission rate of 62 per cent was obtained. Hung, Wilkins, and Blizzard ${ }^{\top}$ reported that successful medical treatment was accomplished in 71 per cent of the patients followed for at least 2 years. The most recently reported series is that of Root and co-workers. ${ }^{8}$ Only 35 per cent of their patients with adequate follow-up were considered to have had satisfactory results.

When the data from these three studies are combined, an over-all cure rate of 57 per cent is obtained. If the criteria are relaxed and a cure is allowed for patients in remission for any length of time following cessation of therapy, 
the incidence of successful treatment becomes 64 per cent. Wilkins ${ }^{9}$ suggests that a cure rate of 50 to 75 per cent can be expected from drug therapy, but we tend to agree with Reeve ${ }^{10}$ and Danowski ${ }^{11}$ that the higher figures are probably optimistic.

Treatment with propylthiouracil and other blocking agents may be complicated by drug reactions such as nausea, dermatitis, urticaria, and leukopenia. Agranulocytosis may occur, but is fortunately rare ( 1 in 500 ).

The operative approach has been faulted mostly because a high incidence of failure to produce a euthyroid state has been reported. A recent report from the Mayo Clinic $^{12}$ documented a 10 per cent incidence of recurrent hyperthyroidism and 38 per cent of hypothyroidism following bilateral subtotal thyroidectomy in 304 children. The frequency of myxedema ranges from 29-50 per cent in other large series. ${ }^{13-10}$

The mortality and morbidity of the surgical procedure must be considered. Only an absence of mortality can compete with drug therapy. Permanent sequelae of the operative procedure especially hypoparathyroidism must be limited. The psychic trauma of hospitalization and operation must be weighed against the inconvenience and higher cost of repeated out-patient visits.

\section{Summary AND Conclusions}

Experience with bilateral subtotal thyroidectomy as the primary form of treatment of thyrotoxicosis in children has been reviewed. The low incidence of postoperative hypothyroidism ( 5 per cent), the lack of recurrent hyperthyroidism, coupled with the low complication rate and absence of mortality has allowed us to advance this as the treatment of choice.

\section{SUMMARIO IN INTERLINGUA}

Es reportate un basse incidentia de complicationes e le complete absentia de mortalitate operatori pro un gruppo de 35 juveniles subjicite a bilateral thyroidectomia subtotal como therapia de thyrotoxicosis. Iste resultatos, insimul con le observate nonrecurrentia de hyperthyroidismo e le incidentia de solmente 5 pro cento de hypothyroidismo documentate post un prolongate periodo de observation postoperatori, convince nos que le intervention chirurgic es le tractamento de election in juveniles con thyrotoxicosis.

\section{REFERENCES}

1. Bacon, G. E., and Lowrey, G. H.: Experience with surgical treatment of thyrotoxicosis in children. J. Pediat. 67:1-5, 1965.

2. Coller, F. A., and Boyden, A. M.: The devclopment of the technique of thyroidectomy. Surg. Gynec. Obstet. 65:495, 1937.

3. Elsas, L. J., Whittemore, R., and Durrow, G. N.: Maternal and neonatal graves disease. J.A.M.A. 200:250, 1967.

4. Starr, P., Jaffe, H. L., and Oettinger, L., Jr.: Late results of $\mathrm{I}^{131}$ treatment of hyperthyroidism in seventy-three children and adolescents. J. Nucl. Med. 5:81, 1964.

5. Solomon, D. H., Beck, J. C., VanderLaan, W. P., and Astwood, E. B.: Prognosis of hyperthyroidism treated with antithyroid drugs. J.A.M.A. 152:201, 1953.

6. Kunstadter, R. H., and Stein, A. F.: Treatment of thyrotoxic children with thiourea derivatives. Amer. J. Dis. Child. $90: 373,1955$.

7. Hung, W., Wilkins, L., and Blizzard, R.: Medical therapy of thyrotoxicosis in children. Pediatrics 30:17, 1962.

8. Root, W. W., Bongiovanni, A. A., Harvie, F. F., and Eberlein, W.: Treatment of juvenile thyrotoxicosis. J. Pediat. 63:402, 1963.

9. Wilkins, L.: The Diagnosis and Treatment of Endocrine Disorders in Childhood 
and Adolescence, ed. 3. Springfield, Ill., Charles C Thomas, 1965.

10. Reeve, T. S.: Current trends in the management of thyroid disease. Med. J. Australia 2:248, 1961.

11. Danowski, T. S.: Clinical Endocrinology, Vol. II: Thyroid. Baltimore, Williams and Wilkins Co., 1962.

12. Hayles, A. B., Enrique, C., and McConahey, W. M.: The treatment of hyperthyroidism in children. Mayo Clinic Proc. $42: 218,1967$.
13. McClintock, J. C., Frawley, R. F., and Holden, J.H.P.: Hyperthyroidism in children. J. Clin. Endocrin. 16:62, 1956 .

14. Arnold, M. B., Talbot, N. B., and Cope, $\mathrm{O}$.: Concerning the choice of therapy for childhood hyperthyroidism. Pediatrics 21:47, 1958.

15. Crawford, J. D.: Goiters in childhood. Pediatrics 17:440, 1956.

16. Kunstadter, R. H., and Stein, A. F.: Treatment of hyperthyroidism in children. Amer. J. Dis. Child. 76:424, 1948. 